\title{
Avaliação da coordenação do cuidado de usuários com tuberculose multidrogarresistente em Recife, Pernambuco, Brasil
}

\author{
Evaluation of care coordination of users with multi-drug tuberculosis \\ in the city of Recife, Pernambuco State, Brazil
}

Denise Bezerra Marinho Barros', Juliana Martins Barbosa da Silva Costa2, Yluska Almeida Coelho dos Reis ${ }^{\mathbf{3}}$, Ana Coelho de Albuquerque', Eronildo Felisberto $\mathbf{1}$

DOI: 10.1590/0103-1104202012407

1 Instituto de Medicina Integral Professor Fernando Figueira (Imip) - Boa Vista (PE), Brasil.

barrosdenise27@gmail.com

2 Universidade Federal de Pernambuco (UFPE), Centro Acadêmico do Agreste (CAA) - Recife (PE), Brasil.

3 Secretaria de Saúde do Estado de Pernambuco (SES-PE), Secretaria Executiva de Vigilância em Saúde (SEVS) - Recife (PE), Brasil.
RESUMO Trata-se de um estudo avaliativo de corte transversal que objetivou avaliar a coordenação do cuidado entre níveis de atenção ao paciente com tuberculose multidrogarresistente em Recife, Pernambuco. A pesquisa foi realizada em três etapas: desenho do modelo lógico, elaboração da matriz de julgamento e análise normativa. Entrevistaram-se médicos e enfermeiros dos três níveis de atenção, bem como gestores do Programa de Controle da Tuberculose. Atribuiu-se pontuação a cada indicador da matriz de julgamento, sendo classificado como excelente ( $\geq 75,0 \%)$, bom (50,0-74,0\%), ruim (49,0\%-25,0\%) ou crítico (<25,0\%). A coordenação do cuidado entre níveis de atenção foi considerada ruim (32,5\%), assim como seus componentes coordenação administrativa (41,2\%), da informação (32,0\%) e da gestão clínica (31,0\%). Percebeu-se que os níveis de atenção se organizam de forma separada, com falhas na interligação da rede de serviços, resultando na assistência descoordenada e fragmentada.

PALAVRAS-CHAVE Avaliação em saúde. Tuberculose. Tuberculose resistente a múltiplos medicamentos. Atenção à saúde.

ABSTRACT This is a cross-sectional evaluative study aimed at evaluating the coordination of care between levels of attention to patients with multidrug-resistant tuberculosis in the city of Recife, State of Pernambuco. It was carried out in three stages: intervention design, elaboration of the judgment matrix and normative analysis. Also physicians and nurses from the three levels of care were interviewed as managers of the Tuberculosis Control Program. Values were assigned to each indicator of the judgment matrix, which was classified as excellent $(\geq 75 \%)$, good (50\% -74\%), poor (49\%-25\%) and critical $(<25 \%)$. Coordination of care between care levels was considered poor (32.5\%), as were administrative coordination (41.2\%), information (32.0\%) and clinical management (31.0\%). It was revealed that the levels of attention are organized separately, with failures in the interconnection of the service network, resulting in uncoordinated and fragmented assistance.

KEYWORDS Health evaluation. Tuberculosis. Tuberculosis resistant to multiple medicines. Health care. 


\section{Introdução}

Os sistemas de saúde dos países da América Latina são caracterizados por altos níveis de fragmentação nos seus serviços, dificultando aspectos como a constituição de redes integradas $\mathbf{1}$, que têm como um dos atributos a existência de mecanismos da coordenação do cuidado ${ }^{2}$. Assim, coordenação do cuidado pode ser definida como a articulação entre os diversos serviços e ações de saúde prestados a determinado paciente, que, independentemente do local onde sejam disponibilizados, operem de forma sincronizada e voltada ao alcance de um objetivo comum ${ }^{3,1}$. Para Starfield4, a essência da coordenação é a disponibilidade de informações a respeito de problemas e serviços anteriores e o reconhecimento dessa informação, na medida em que está relacionada às necessidades do atendimento. Essa coordenação pode ser dividida em coordenação da informação, coordenação da gestão clínica e coordenação administrativa ${ }^{5,6}$.

A tuberculose (TB) é uma condição crônica e continua sendo um importante problema de saúde mundial. Dentre seus maiores problemas ao longo dos anos um dos mais preocupantes e discutidos tem sido o fenômeno da multirresistência ${ }^{7}$. No Brasil, considera-se caso de tuberculose multidrogarresistente (TBMDR) aquele com diagnóstico confirmado por cultura e identificação de Mycobacterium tuberculosis e por teste de sensibilidade com resistência à rifampicina e isoniazida e a mais um terceiro fármaco dos esquemas padroniza$\operatorname{dos}^{6,7}$. A resistência às drogas está associada à ineficiência dos serviços de saúde no que diz respeito principalmente à capacidade de detecção precoce de casos novos e dos desfechos desfavoráveis como as falências e o abandono ao tratamento. Essa forma da tuberculose exige assistência mais complexa, pois esses pacientes necessitam de gerenciamento para manejo e cuidado ao longo do tempo, ou seja, de continuidade da assistência, de forma a garantir a adesão e o tratamento em longo prazo ${ }^{6,8}$.

Dentre os problemas para o seu controle está também a organização dos serviços de saúde para detectar e tratar os casos. Por ser uma doença de grande transmissibilidade, além de condição crônica, seu acompanhamento requer monitoramento constante e a atenção deve ser desenvolvida de modo integrado. Ela deve ser tratada e acompanhada em centros de referência por profissionais especializados e por equipe multidisciplinar, com articulação entre todos os níveis de atenção, para que haja controle efetivo da doença ${ }^{6,7}$. Portanto a coordenação é fundamental nesse processo, pois garante a articulação entre os diferentes serviços e profissionais envolvidos na assistência aos pacientes $\mathbf{1 , 4}$.

Percebe-se que os serviços de saúde que prestam assistência aos usuários com TB-MDR apresentam problemas com os mecanismos de coordenação, pois há descontinuidade do fluxo de informação e inexistência de um sistema que os integre e otimize o seguimento do paciente, comprometendo o cuidado7. Um sistema de atenção centralizado apresenta dificuldade em proporcionar cuidado continuado a esses usuários, resultando no insucesso do tratamento ${ }^{9,10}$. Isso, porque o modelo de atendimento do SUS é baseado na hierarquização das ações e serviços por níveis de complexidade e acontece em três níveis de atenção de diferentes densidades tecnológicas ${ }^{5}$.

Assim, admitindo que uma coordenação eficaz pode proporcionar assistência adequada aos usuários com TB-MDR 11, este estudo tem como objetivo avaliar a coordenação do cuidado entre os níveis de atenção ao paciente com TB-MDR na rede de serviços de saúde do município de Recife, Pernambuco.

\section{Material e métodos}

\section{Delineamento}

Pesquisa avaliativa de corte transversal, que adotou o estudo de caso único como meio de investigação ${ }^{12}$; o município de Recife foi eleito 
como unidade de análise e o estudo de caso investigou a coordenação do cuidado entre os níveis de atenção ao paciente com TB-MDR.

\section{Contexto}

A pesquisa foi realizada em Recife, capital pernambucana, município que, ao longo dos últimos anos, tem apresentado resultados preocupantes no que diz respeito aos indicadores de tuberculose. Em 2016, apresentou a maior taxa de mortalidade (6,4/100 mil hab.), e, em 2017, registrou a terceira maior incidência (85,5/100 mil hab.) entre as capitais brasileiras $^{13}$. Com relação à atenção básica, a cobertura estimada é de $73,0 \%$, sendo $59,0 \%$ de Estratégia Saúde da Família (ESF), composta por 130 unidades e 276 equipes, e 14,0\% de Estratégia de Agente Comunitário de Saúde (EACS) ${ }^{14}$.

\section{Etapas do processo avaliativo}

O estudo foi realizado em três etapas:

\section{ETAPA 1 - DESENHO DO MODELO LÓGICO}

O desenho do modelo lógico detalhou os componentes e permitiu a visualização esquemática do funcionamento da coordenação do cuidado entre os níveis de atenção ao paciente com tuberculose multidrogarresistente ${ }^{13}$. Sua elaboração atendeu especificamente este estudo e utilizou como base teórica as seguintes referências: Reid et al. ${ }^{15}$, Vázquez et al.', Mendes ${ }^{\mathbf{2}}$, Starfield ${ }^{\mathbf{3}}$ e o Manual de Recomendações para Controle da Tuberculose no Brasil5. Para validação, foi submetido à consulta com sete especialistas, profissionais e gestores do Programa Nacional e Estadual de Controle da Tuberculose e acadêmicos envolvidos em estudos sobre a coordenação do cuidado, que verificaram a completude dos elementos e a consistência das relações causais, tendo como base a técnica denominada 'Conferência de Consenso'16, adaptada para os fins da pesquisa. A consulta foi realizada por e-mail, videoconferência e de modo presencial, de acordo com a disponibilidade e preferência do especialista.

O modelo apresenta três componentes e seus respectivos subcomponentes: coordenação da informação, composta dos subcomponentes registro da informação, transferência da informação e utilização da informação; coordenação da gestão clínica, formada pelos subcomponentes seguimento do paciente e coerência da atenção; e coordenação administrativa (figura 1). 
Figura 1. Modelo lógico da coordenação do cuidado entre os níveis de atenção ao paciente com tuberculose multidrogarresistente

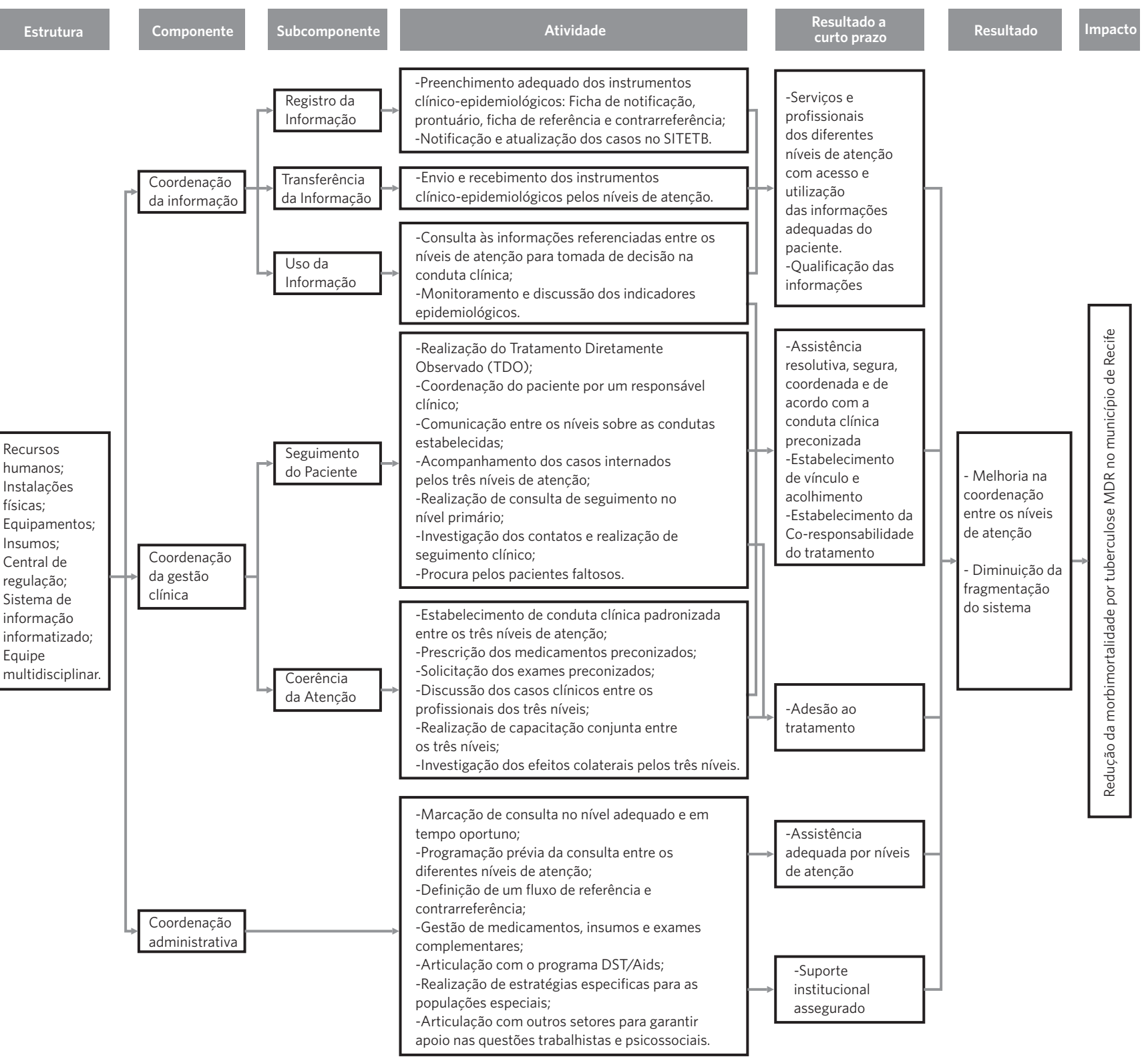

\section{ETAPA 2 - ELABORAC̣ÃO DA MATRIZ DE JULGAMENTO}

Após o desenho do modelo lógico, procedeu-se à elaboração da matriz de julgamento. $\mathrm{O}$ quadro 1 apresenta, para cada um dos componentes do modelo lógico, indicadores, parâmetros adotados para o julgamento, fontes de verificação e a técnica de obtenção que guiou a coleta de dados. 
Quadro 1. Matriz de julgamento da coordenação do cuidado entre os níveis de atenção ao paciente com tuberculose multidrogarresistente

\begin{tabular}{|c|c|c|c|c|c|c|}
\hline $\begin{array}{l}\text { Compo- } \\
\text { nente }\end{array}$ & $\begin{array}{l}\text { Subcompo- } \\
\text { nente }\end{array}$ & Indicador & Pontuação & $\begin{array}{l}\text { Pontuação } \\
\text { máxima }\end{array}$ & $\begin{array}{l}\text { Fonte de } \\
\text { dados }\end{array}$ & $\begin{array}{l}\text { Técnica de ob- } \\
\text { tenção }\end{array}$ \\
\hline \multirow{11}{*}{ 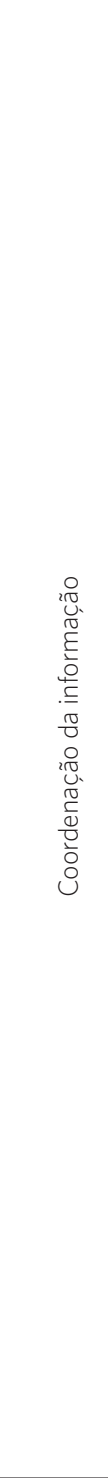 } & \multirow[t]{6}{*}{$\begin{array}{l}\text { Registro da } \\
\text { informação }\end{array}$} & $\begin{array}{l}\text { Existência dos instrumentos clínico epide- } \\
\text { miológico, como ficha de notificação, ficha de } \\
\text { referência e contrarreferência }\end{array}$ & $\begin{array}{l}\text { Existência das duas fichas = } \\
0,75 ; \text { Existência de uma das } \\
\text { fichas }=0,5 ; \text { Inexistência das } \\
\text { duas fichas = } 0\end{array}$ & 0,75 & Secundário & $\begin{array}{l}\text { Ficha de noti- } \\
\text { ficação e ficha } \\
\text { de referência e } \\
\text { contrarreferência }\end{array}$ \\
\hline & & $\begin{array}{l}\text { \% profissionais preenchendo o prontuário } \\
\text { do paciente com as informações necessárias } \\
\text { sobre diagnóstico, motivo da referência, indica- } \\
\text { ções para o seguimento da atenção, comorbi- } \\
\text { dades, resultados e exames solicitados, trata- } \\
\text { mentos farmacológicos, situação psicossocial, } \\
\text { outras }\end{array}$ & $\begin{array}{l}\geq 80 \%=0,75 ; 60 \%-79 \%= \\
0,5 ;<60 \%=0\end{array}$ & 0,75 & Secundário & Prontuário \\
\hline & & $\begin{array}{l}\text { \% profissionais preenchendo adequadamente a } \\
\text { ficha de notificação do paciente }\end{array}$ & $\begin{array}{l}\geq 80 \%=0,75 ; 60 \%-79 \%= \\
0,5 ;<60 \%=0\end{array}$ & 0,75 & Secundário & $\begin{array}{l}\text { Ficha de notifi- } \\
\text { cação }\end{array}$ \\
\hline & & $\begin{array}{l}\text { \% profissionais preenchendo a ficha de refe- } \\
\text { rência e contrarreferência com as informações } \\
\text { necessárias, como diagnóstico, motivo da } \\
\text { referência, indicações para o seguimento da } \\
\text { atenção, comorbidades, resultados e exames } \\
\text { solicitados, tratamentos farmacológicos, situa- } \\
\text { ção psicossocial, outras }\end{array}$ & $\begin{array}{l}\geq 80 \%=1 ; 60 \%-79 \%=0,5 \\
<60 \%=0\end{array}$ & 1 & Secundário & $\begin{array}{l}\text { Ficha de referên- } \\
\text { cia e contrarrefe- } \\
\text { rência }\end{array}$ \\
\hline & & $\begin{array}{l}\% \text { profissionais preenchendo o resumo de alta } \\
\text { hospitalar }\end{array}$ & $\begin{array}{l}\geq 80 \%=1 ; 60 \%-79 \%=0,5 \\
<60 \%=0\end{array}$ & 1 & Primário & Entrevista \\
\hline & & Alimentação do SITETB de forma oportuna & $\operatorname{SIM}=0,75 \mathrm{NA} O=0$ & 0,75 & Secundário & SITETB \\
\hline & \multirow[t]{3}{*}{$\begin{array}{l}\text { Transferência } \\
\text { da informa- } \\
\text { ção }\end{array}$} & $\begin{array}{l}\text { \% profissionais enviando a referência e con- } \\
\text { trarreferência para os diferentes níveis de } \\
\text { atenção }\end{array}$ & $\begin{array}{l}\geq 80 \%=3 ; 60 \%-79 \%=1,5 \\
<60 \%=0\end{array}$ & 3 & Primário & Entrevista \\
\hline & & $\begin{array}{l}\text { \% profissionais recebendo a referência e con- } \\
\text { trarreferência dos diferentes níveis de atenção }\end{array}$ & $\begin{array}{l}\geq 80 \%=4 ; 60 \%-79 \%=2 \\
<60 \%=0\end{array}$ & 4 & Primário & Entrevista \\
\hline & & $\begin{array}{l}\text { \% profissionais enviando o resumo de alta } \\
\text { hospitalar para os diferentes níveis de atenção }\end{array}$ & $\begin{array}{l}\geq 80 \%=3 ; 60 \%-79 \%=1,5 \\
<60 \%=0\end{array}$ & 3 & Primário & Entrevista \\
\hline & \multirow[t]{2}{*}{$\begin{array}{l}\text { Uso da infor- } \\
\text { mação }\end{array}$} & $\begin{array}{l}\text { \% profissionais utilizando as informações } \\
\text { referenciadas entre os níveis de atenção para a } \\
\text { tomada de decisão na conduta clínica }\end{array}$ & $\begin{array}{l}\geq 80 \%=5 ; 60 \%-79 \%=2,5 \\
<60 \%=0\end{array}$ & 5 & Primário & Entrevista \\
\hline & & $\begin{array}{l}\text { Percentual de profissionais dos três níveis de } \\
\text { atenção monitorando e discutindo os indicado- } \\
\text { res epidemiológicos }\end{array}$ & $\begin{array}{l}\geq 80 \%=5 ; 60 \%-79 \%=2,5 \\
<60 \%=0\end{array}$ & 5 & Primário & Entrevista \\
\hline \multirow{3}{*}{ 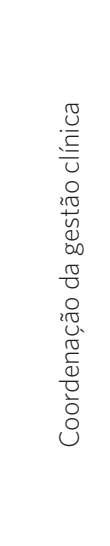 } & \multirow{3}{*}{$\begin{array}{l}\text { Seguimento } \\
\text { do paciente }\end{array}$} & Realização do TDO & $\mathrm{SIM}=3 ; \mathrm{NA} O \mathrm{O}=0$ & 3 & Primário & Entrevista \\
\hline & & $\begin{array}{l}\text { Existência de uma equipe multidisciplinar } \\
\text { no nível terciário composta por enfermeiro, } \\
\text { médico, assistente social, técnico de enferma- } \\
\text { gem, farmacêutico, bioquímico, nutricionista, } \\
\text { psicólogo }\end{array}$ & $\begin{array}{l}\text { Equipe multidisciplinar } \\
\text { completa= } 3 \text { Equipe mul- } \\
\text { tidisciplinar com apenas } \\
\text { médico, enfermeiro, técnico } \\
\text { de enfermagem e assistente } \\
\text { social = } 1,5 \text { Equipe multi- } \\
\text { disciplinar sem pelo menos } \\
\text { médico, enfermeiro, técnico } \\
\text { de enfermagem e assistente } \\
\text { social = } 0\end{array}$ & 3 & Primário & Entrevista \\
\hline & & $\begin{array}{l}\text { Existência de um responsável clínico para } \\
\text { coordenar o seguimento do paciente entre os } \\
\text { níveis de atenção }\end{array}$ & $\mathrm{SIM}=4 ; \mathrm{NA} O=0$ & 4 & Primário & Entrevista \\
\hline
\end{tabular}


Quadro 1. (cont.)

\begin{tabular}{|c|c|c|c|c|c|c|}
\hline $\begin{array}{l}\text { Compo- } \\
\text { nente }\end{array}$ & $\begin{array}{l}\text { Subcompo- } \\
\text { nente }\end{array}$ & Indicador & Pontuação & $\begin{array}{l}\text { Pontuação } \\
\text { máxima }\end{array}$ & $\begin{array}{l}\text { Fonte de } \\
\text { dados }\end{array}$ & $\begin{array}{l}\text { Técnica de ob- } \\
\text { tenção }\end{array}$ \\
\hline \multirow{5}{*}{ 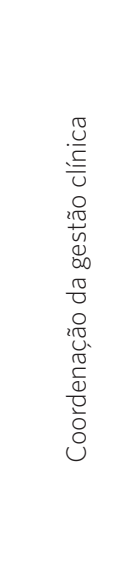 } & \multirow[t]{5}{*}{$\begin{array}{l}\text { Seguimento } \\
\text { do paciente }\end{array}$} & $\begin{array}{l}\text { \% profissionais médicos do primeiro nível } \\
\text { que consultam o médico especialista para } \\
\text { esclarecer as dúvidas sobre o seguimento dos } \\
\text { pacientes }\end{array}$ & $\begin{array}{l}\geq 80 \%=3 ; 60 \%-79 \%=1,5 \\
<60 \%=0\end{array}$ & 3 & Primário & Entrevista \\
\hline & & $\begin{array}{l}\text { \% profissionais médicos da atenção primária } \\
\text { que realizam a consulta de seguimento do } \\
\text { paciente TB-MDR }\end{array}$ & $\begin{array}{l}\geq 80 \%=3 ; 60 \%-79 \%=1,5 \\
<60 \%=0\end{array}$ & 3 & Primário & Entrevista \\
\hline & & $\begin{array}{l}\text { \% profissionais dos três níveis de atenção reali- } \\
\text { zando acompanhamento dos casos internados }\end{array}$ & $\begin{array}{l}\geq 80 \%=3 ; 60 \%-79 \%=1,5 \\
<60 \%=0\end{array}$ & 3 & Primário & Entrevista \\
\hline & & $\begin{array}{l}\text { \% profissionais dos três níveis de atenção que } \\
\text { investigam os contatos e realizam seguimento } \\
\text { clínico }\end{array}$ & $\begin{array}{l}\geq 80 \%=3 ; 60 \%-79 \%=1,5 \\
<60 \%=0\end{array}$ & 3 & Primário & Entrevista \\
\hline & & $\begin{array}{l}\text { \% profissionais dos três níveis de atenção que } \\
\text { realizam busca dos faltosos }\end{array}$ & $\begin{array}{l}\geq 80 \%=3 ; 60 \%-79 \%=1,5 \\
<60 \%=0\end{array}$ & 3 & Primário & Entrevista \\
\hline \multirow{8}{*}{ 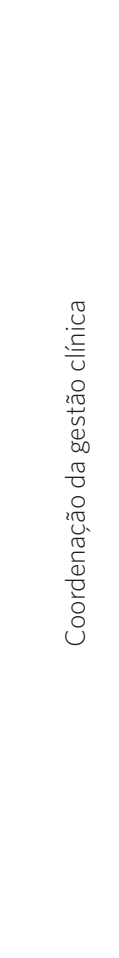 } & \multirow[t]{8}{*}{$\begin{array}{l}\text { Coerência da } \\
\text { atenção }\end{array}$} & $\begin{array}{l}\text { \% pacientes com medicamentos preconizados } \\
\text { disponíveis para o tratamento da TB-MDR }\end{array}$ & $\begin{array}{l}\geq 80 \%=3 ; 60 \%-79 \%=1,5 \\
<60 \%=0\end{array}$ & 3 & Secundário & SITETB \\
\hline & & $\begin{array}{l}\text { \% pacientes realizando todos os exames } \\
\text { preconizados, como baciloscopia de escarro, } \\
\text { cultura de secreção, raio-x, teste anti-HIV, para } \\
\text { o diagnóstico e acompanhamento da TB-MDR }\end{array}$ & $\begin{array}{l}\geq 80 \%=3 ; 60 \%-79 \%=1,5 \\
<60 \%=0\end{array}$ & 3 & Secundário & SITETB \\
\hline & & $\begin{array}{l}\text { Existência de protocolo padronizado entre os } \\
\text { níveis para o acompanhamento e tratamento } \\
\text { do paciente TB-MDR }\end{array}$ & $\mathrm{SIM}=3 ; \mathrm{NA} O=0$ & 3 & Primário & Entrevista \\
\hline & & $\begin{array}{l}\text { \% profissionais que conhecem e utilizam } \\
\text { protocolo para a conduta clínica, como diag- } \\
\text { nóstico, tratamento, exames, critérios de } \\
\text { encaminhamento para outro nível de atenção, } \\
\text { entre níveis }\end{array}$ & $\begin{array}{l}\geq 80 \%=3 ; 60 \%-79 \%=1,5 \\
<60 \%=0\end{array}$ & 3 & Primário & Entrevista \\
\hline & & $\begin{array}{l}\text { Percentual de profissionais realizando discus- } \\
\text { são dos casos clínicos entre os diferentes níveis } \\
\text { de atenção }\end{array}$ & $\begin{array}{l}\geq 80 \%=4 ; 60 \%-79 \%=2 \\
<60 \%=0\end{array}$ & 4 & Primário & Entrevista \\
\hline & & $\begin{array}{l}\text { Percentual de profissionais realizando matricia- } \\
\text { mento dos casos entre os níveis de atenção }\end{array}$ & $\begin{array}{l}\geq 80 \%=3 ; 60 \%-79 \%=1,5 \\
<60 \%=0\end{array}$ & 3 & Primário & Entrevista \\
\hline & & $\begin{array}{l}\text { Realização de capacitação conjunta entre os } \\
\text { profissionais dos diferentes níveis de atenção }\end{array}$ & $\mathrm{SIM}=3 ; N \tilde{A} O=0$ & 3 & Primário & Entrevista \\
\hline & & $\begin{array}{l}\text { Percentual de profissionais dos três níveis de } \\
\text { atenção investigando os efeitos colaterais }\end{array}$ & $\begin{array}{l}\geq 80 \%=3 ; 60 \%-79 \%=1,5 \\
<60 \%=0\end{array}$ & 3 & Primário & Entrevista \\
\hline $\begin{array}{l}\text { Compo- } \\
\text { nente }\end{array}$ & \multicolumn{2}{|l|}{ Indicador } & Pontuação & $\begin{array}{l}\text { Pontuação } \\
\text { máxima }\end{array}$ & $\begin{array}{l}\text { Fonte de } \\
\text { dados }\end{array}$ & $\begin{array}{l}\text { Técnica de ob- } \\
\text { tenção }\end{array}$ \\
\hline \multirow{3}{*}{ 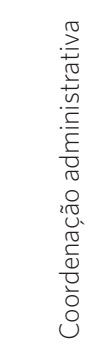 } & \multicolumn{2}{|c|}{$\begin{array}{l}\text { Existência de fluxos assistenciais definidos para o paciente } \\
\text { TB-MDR }\end{array}$} & $\mathrm{SIM}=3 ; \mathrm{NA} O=0$ & 3 & Primário & Entrevista \\
\hline & \multicolumn{2}{|c|}{$\begin{array}{l}\text { Existência de leitos com isolamento respiratório em quanti- } \\
\text { dade suficiente }\end{array}$} & $\mathrm{SIM}=2 ; \mathrm{NA} O=0$ & 2 & Primário & Entrevista \\
\hline & \multicolumn{2}{|c|}{$\begin{array}{l}\text { \% unidades de saúde com adequada estrutura de biossegu- } \\
\text { rança, como sala de atendimento com mesa de escritório, } \\
\text { cadeiras para o usuário e o acompanhante e mesa de exame } \\
\text { clínico; lavatório com torneiras; ambientes claros, com lumi- } \\
\text { nosidade natural se possível; janelas ou ventilação indireta }\end{array}$} & $\begin{array}{l}\geq 80 \%=2 ; 60 \%-79 \%= \\
1 ;<60 \%=0\end{array}$ & 2 & Primário & Entrevista \\
\hline
\end{tabular}


Quadro 1. (cont.)

\begin{tabular}{|c|c|c|c|c|c|}
\hline $\begin{array}{l}\text { Compo- } \\
\text { nente }\end{array}$ & Indicador & Pontuação & $\begin{array}{l}\text { Pontuação } \\
\text { máxima }\end{array}$ & $\begin{array}{l}\text { Fonte de } \\
\text { dados }\end{array}$ & $\begin{array}{l}\text { Técnica de ob- } \\
\text { tenção }\end{array}$ \\
\hline \multirow{10}{*}{ 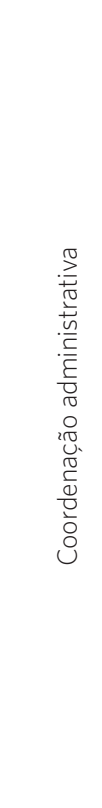 } & $\begin{array}{l}\text { adequada (exaustores), possibilitando a circulação de ar, } \\
\text { para atendimento aos pacientes TB-MDR }\end{array}$ & & & & \\
\hline & $\begin{array}{l}\text { Funcionamento adequado dos fluxos assistenciais de refe- } \\
\text { rência e contrarreferência definidos para o paciente TB-MDR }\end{array}$ & $\mathrm{SIM}=2 ; \mathrm{NA} O=0$ & 2 & Primário & Entrevista \\
\hline & $\begin{array}{l}\text { Existência de consultas marcadas no nível adequado em } \\
\text { tempo oportuno }\end{array}$ & $\mathrm{SIM}=2 ; \mathrm{NA} O=0$ & 2 & Primário & Entrevista \\
\hline & $\begin{array}{l}\text { Existência de consultas marcadas com programação prévia } \\
\text { para os diferentes níveis de atenção }\end{array}$ & $\mathrm{SIM}=2 ; \mathrm{NA} O=0$ & 2 & Primário & Entrevista \\
\hline & Realização do controle logístico dos medicamentos & $\mathrm{SIM}=2 ; \mathrm{NA} O \mathrm{O}=0$ & 2 & Primário & Entrevista \\
\hline & Realização do controle logístico dos insumos & $\mathrm{SIM}=2 ; \mathrm{NA} O=0$ & 2 & Primário & Entrevista \\
\hline & Realização do controle logístico dos exames & $\mathrm{SIM}=2 ; \mathrm{NA} O \mathrm{O}=0$ & 2 & Primário & Entrevista \\
\hline & Articulação e integração com o Programa de DST/Aids & $\mathrm{SIM}=2 ; \mathrm{NA} O=0$ & 2 & Primário & Entrevista \\
\hline & $\begin{array}{l}\text { Identificação das melhores estratégias para a abordagem } \\
\text { das populações com maior vulnerabilidade de adoecimen- } \\
\text { to: população privada de liberdade, população vivendo em } \\
\text { situação de rua, povos indígenas }\end{array}$ & $\mathrm{SIM}=2 ; \mathrm{NA} O=0$ & 2 & Primário & Entrevista \\
\hline & $\begin{array}{l}\text { Articulação com outros setores para garantir apoio trabalhis- } \\
\text { ta e psicossocial aos pacientes }\end{array}$ & $\mathrm{SIM}=2 ; \mathrm{NA} O=0$ & 2 & Primário & Entrevista \\
\hline
\end{tabular}

Fonte: Elaboração própria.

Os indicadores elencados na matriz foram calculados a partir de dados obtidos de entrevistas realizadas com profissionais e gestores envolvidos na assistência aos pacientes com TB-MDR. Inicialmente, foram identificados no Sistema de Informação de Tratamentos Especiais da Tuberculose (SITETB) os casos que em 2014 receberam tratamento, totalizando 21 pacientes. Posteriormente, entrevistaram-se os médicos e enfermeiros dos três níveis de atenção que realizaram o atendimento e o acompanhamento desses casos. No âmbito municipal, esses pacientes foram atendidos e acompanhados por $15 \mathrm{ESF}$, três EACS e três unidades especializadas. No âmbito estadual, todos eles foram atendidos e acompanhados pelo hospital de referência terciária. Além dos serviços de atendimento ao paciente com TB-MDR, foram entrevistados os coordenadores distritais do município de Recife e o coordenador estadual do Programa de Controle da Tuberculose (PCT).
Para a coleta de dados primários, foram aplicados dois questionários estruturados, sendo um para os médicos e enfermeiros dos três níveis de atenção e o outro para os coordenadores distritais e o coordenador estadual do PCT. Realizou-se estudo piloto em uma unidade de referência secundária de um município da região metropolitana de Recife para o pré-teste do instrumento. Os dados secundários foram coletados do SITETB da Secretaria Executiva de Vigilância em Saúde, Secretaria Estadual de Saúde de Pernambuco (SEVS, SES-PE). As entrevistas foram realizadas no período de maio a setembro de 2015 .

\section{ETAPA 3 - CLASSIFICAC̣ÃO DO GRAU DE IMPLANTAC̣ÃO}

Após a coleta, foi construído um banco de dados no Microsoft Excel. Cada componente da matriz recebeu uma pontuação a partir do número de indicadores que a compõe e da 
importância que cada um dos componentes guarda com a coordenação do cuidado, identificado por meio da revisão de literatura. Assim, a coordenação da informação e a coordenação administrativa receberam 25 pontos cada, e a coordenação da gestão clínica, 50 pontos, totalizando 100 pontos. A pontuação dos componentes foi distribuída entre os indicadores e considerou a relação de importância de cada um com o componente avaliado, também identificada por meio da revisão teórica. $\mathrm{O}$ resultado de cada indicador foi comparado com um padrão ou norma. Para os indicadores cujo padrão foi a existência ou realização de um determinado item ou ação, a pontuação final foi dada pela média advinda do somatório das pontuações verificadas em cada entrevista dividido pelo número de entrevistados.

Já os indicadores cujo padrão foi o percentual de profissionais que realizaram a ação, a pontuação final do indicador foi dada pela média advinda do número de profissionais que realizaram a ação dividido pelo número de profissionais entrevistados. O resultado foi confrontado com o padrão estabelecido pela matriz de julgamento, sendo aplicada a pontuação correspondente a cada indicador analisado. Quando o indicador obteve $\geq 80 \%$ de respostas positivas, recebeu a pontuação máxima de $60-79 \%$ a metade da pontuação e menor que $60 \%$, zero pontos (quadro 1 ).

O julgamento de valor foi realizado por meio do cálculo de uma regra de três simples para determinar a situação da coordenação do cuidado entre os níveis de atenção ao paciente TB-MDR. A pontuação obtida foi dividida pela pontuação máxima e multiplicado por 100. Para a classificação, utilizou-se o corte por quartis: implantado ( $\geq 75,0 \%)$, implantação parcial (50,0\%-74,9\%), implantação incipiente (49,9\%-25,0\%) e não implantado (menor que $24,9 \%$ ).

\section{Aspectos éticos}

O projeto foi submetido e aprovado pelo Comitê de Ética em Pesquisa em Seres Humanos do Instituto de Medicina Integral Professor Fernando Figueira (Imip) sob o registro 4688-15 e seguiu as recomendações da Resolução do Conselho Nacional de Saúde $n^{0}$ 466/12 e complementares.

\section{Resultados}

A implantação da coordenação do cuidado entre os níveis de atenção ao paciente com TB-MDR no município de Recife foi considerada incipiente $(32,5 \%)$. O componente que apresentou o maior resultado foi a 'coordenação administrativa' (41,2\%), seguido da 'coordenação da informação' (32,0\%) e da 'coordenação da gestão clínica' (31,0\%), classificados como implantação incipiente.

$\mathrm{Na}$ 'coordenação administrativa', apesar de $74,2 \%$ dos profissionais afirmarem que existem fluxos assistenciais definidos para o paciente TB-MDR, apenas $45,2 \%$ relataram que o funcionamento era adequado. Outros aspectos relevantes referem-se à insuficiência de leitos com isolamento respiratório; inexistência de unidades de saúde em todos os níveis de atenção com adequada estrutura de biossegurança para atender aos pacientes TB-MDR; e falta de acesso, por parte dos pacientes em tratamento, a benefícios como vale transporte e cesta básica, apoio trabalhista e psicossocial (quadro 2). 
Quadro 2. Resultado do componente 'coordenação administrativa' entre os níveis de atenção ao paciente com tuberculose multidrogarresistente. Recife, 2015

\begin{tabular}{|c|c|c|c|c|c|}
\hline Componente & Indicador & $\begin{array}{l}\text { Pontuação } \\
\text { Obtida }\end{array}$ & $\begin{array}{l}\text { Pontuação } \\
\text { Máxima }\end{array}$ & $\begin{array}{l}\text { Resultado (\%) e clas- } \\
\text { sificação por indicador }\end{array}$ & $\begin{array}{l}\text { Resultado final (\%) } \\
\text { do componente }\end{array}$ \\
\hline \multirow{12}{*}{ 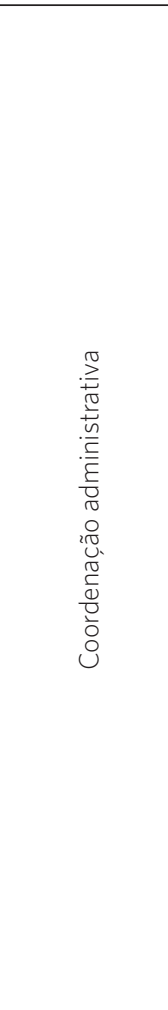 } & $\begin{array}{l}\text { Existência de fluxos assistenciais definidos para o paciente } \\
\text { TB-MDR }\end{array}$ & 2,23 & 3 & $\begin{array}{l}74,2 \\
\text { implantação parcial }\end{array}$ & \multirow{12}{*}{ 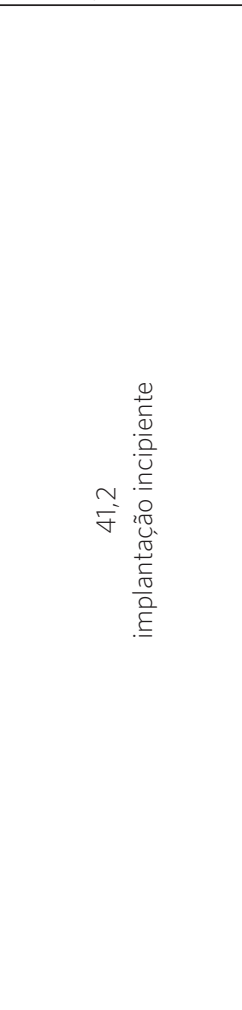 } \\
\hline & $\begin{array}{l}\text { Existência de leitos com isolamento respiratório em quanti- } \\
\text { dade suficiente }\end{array}$ & 0 & 2 & $\begin{array}{l}0,0 \\
\text { não implantado }\end{array}$ & \\
\hline & $\begin{array}{l}\text { \% unidades de saúde com adequada estrutura de biosse- } \\
\text { gurança }\end{array}$ & 0 & 2 & $\begin{array}{l}0,0 \\
\text { não implantado }\end{array}$ & \\
\hline & $\begin{array}{l}\text { Funcionamento adequado dos fluxos assistenciais de } \\
\text { referência e contrarreferência definidos para o paciente } \\
\text { TB-MDR }\end{array}$ & 0,9 & 2 & $\begin{array}{l}45,2 \\
\text { implantação incipiente }\end{array}$ & \\
\hline & $\begin{array}{l}\text { Existência de consultas marcadas no nível adequado em } \\
\text { tempo oportuno }\end{array}$ & 1,61 & 2 & $\begin{array}{l}80,6 \\
\text { implantado }\end{array}$ & \\
\hline & $\begin{array}{l}\text { Existência de consultas marcadas com programação prévia } \\
\text { para os diferentes níveis de atenção }\end{array}$ & 1,74 & 2 & $\begin{array}{l}87,1 \\
\text { implantado }\end{array}$ & \\
\hline & Realização do controle logístico dos medicamentos & 0 & 2 & $\begin{array}{l}0,0 \\
\text { não implantado }\end{array}$ & \\
\hline & Realização do controle logístico dos insumos & 1,33 & 2 & $\begin{array}{l}66,7 \\
\text { implantação parcial }\end{array}$ & \\
\hline & Realização do controle logístico dos exames & 0,67 & 2 & $\begin{array}{l}33,3 \\
\text { implantação incipiente }\end{array}$ & \\
\hline & Articulação e integração com o Programa de DST/Aids & 1,03 & 2 & $\begin{array}{l}51,6 \\
\text { implantação parcial }\end{array}$ & \\
\hline & $\begin{array}{l}\text { Identificação das melhores estratégias para a abordagem } \\
\text { das populações com maior vulnerabilidade de adoecimento }\end{array}$ & 0,77 & 2 & $\begin{array}{l}38,7 \\
\text { implantação incipiente }\end{array}$ & \\
\hline & $\begin{array}{l}\text { Garantia de benefícios, como vale transporte, cesta básica, } \\
\text { apoio trabalhistas e psicossociais aos pacientes em trata- } \\
\text { mento }\end{array}$ & 0 & 2 & $\begin{array}{l}0,0 \\
\text { não implantado }\end{array}$ & \\
\hline
\end{tabular}

Fonte: Elaboração própria.

Entre os subcomponentes da 'coordenação da informação', o que apresentou o melhor resultado foi o 'registro da informação' (70,0\%), seguido de 'transferência da informação' (45,0\%), ambos com implantação parcial; e 'uso da informação' (0,0\%), classificado como não implantado. No subcomponente 'registro da informação', todos os profissionais afirmaram preencher adequadamente a ficha de notificação do paciente e o resumo de alta hospitalar, bem como afirmaram que a alimentação do SITETB se dá de forma oportuna. Por outro lado, nenhum deles preenchia adequadamente a ficha de referência e contrarreferência. No subcomponente 'transferência da informação', apesar de $77,4 \%$ dos profissionais afirmarem enviar a referência e contrarreferência para o outro nível de atenção, apenas $25,8 \%$ afirmaram recebê-la. No subcomponente 'uso da informação', 35,5\% dos respondentes afirmaram utilizar as informações referenciadas entre os níveis de atenção para a tomada de decisão na conduta clínica e apenas $16,1 \%$ dos profissionais dos três níveis de atenção afirmaram monitorar e discutir os indicadores epidemiológicos (quadro 3). 
Quadro 3. Resultado do componente 'coordenação da informação' entre os níveis de atenção ao paciente com tuberculose multidrogarresistente. Recife, 2015

\begin{tabular}{|c|c|c|c|c|c|c|}
\hline Componente & Subcomponente & Indicador & $\begin{array}{l}\text { Pontuação } \\
\text { Obtida }\end{array}$ & $\begin{array}{l}\text { Pontuação } \\
\text { Máxima }\end{array}$ & $\begin{array}{l}\text { Resultado (\%) e clas- } \\
\text { sificação por indicador }\end{array}$ & $\begin{array}{l}\text { Resultado final (\%) } \\
\text { do componente }\end{array}$ \\
\hline \multirow{11}{*}{ 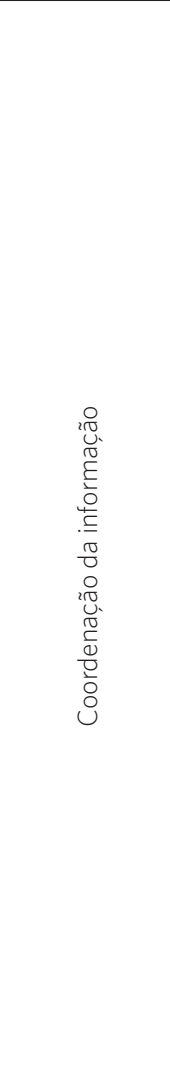 } & \multirow[t]{6}{*}{$\begin{array}{l}\text { Registro da } \\
\text { informação }\end{array}$} & $\begin{array}{l}\text { Existência da ficha de notificação ou da } \\
\text { ficha de referência e contrarreferência }\end{array}$ & 0,50 & 0,75 & $\begin{array}{l}66,7 \\
\text { implantação parcial }\end{array}$ & \multirow{6}{*}{ 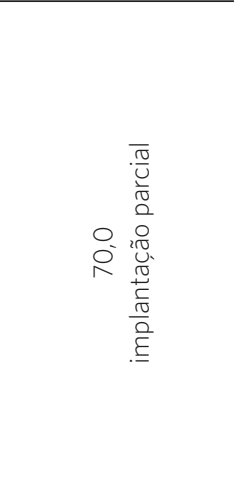 } \\
\hline & & $\begin{array}{l}\text { \% profissionais preenchendo adequada- } \\
\text { mente o prontuário do paciente }\end{array}$ & 0,50 & 0,75 & $\begin{array}{l}66,7 \\
\text { implantação parcial }\end{array}$ & \\
\hline & & $\begin{array}{l}\text { \% profissionais preenchendo adequada- } \\
\text { mente a ficha de notificação do paciente }\end{array}$ & 0,75 & 0,75 & $\begin{array}{l}100,0 \\
\text { implantado }\end{array}$ & \\
\hline & & $\begin{array}{l}\text { \% profissionais preenchendo a ficha de } \\
\text { referência e contrarreferência com as } \\
\text { informações necessárias }\end{array}$ & 0,00 & 1 & $\begin{array}{l}0,0 \\
\text { não implantado }\end{array}$ & \\
\hline & & $\begin{array}{l}\text { \% profissionais preenchendo o resumo } \\
\text { de alta hospitalar }\end{array}$ & 1,00 & 1 & $\begin{array}{l}100,0 \\
\text { implantado }\end{array}$ & \\
\hline & & $\begin{array}{l}\text { Alimentação do SITETB de forma opor- } \\
\text { tuna }\end{array}$ & 0,75 & 0,75 & $\begin{array}{l}100,0 \\
\text { implantado }\end{array}$ & \\
\hline & \multirow[t]{3}{*}{$\begin{array}{l}\text { Transferência da } \\
\text { informação }\end{array}$} & $\begin{array}{l}\text { \% profissionais enviando a referência } \\
\text { e contrarreferência para os diferentes } \\
\text { níveis de atenção }\end{array}$ & 1,50 & 3 & $\begin{array}{l}\text { 50,0 } \\
\text { implantação parcial }\end{array}$ & \multirow{3}{*}{ 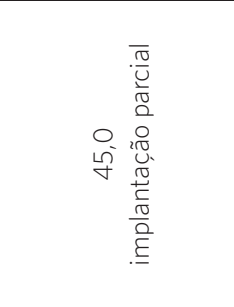 } \\
\hline & & $\begin{array}{l}\text { \% profissionais recebendo a contrarrefe- } \\
\text { rência dos diferentes níveis de atenção }\end{array}$ & 0,00 & 4 & $\begin{array}{l}0,0 \\
\text { não implantado }\end{array}$ & \\
\hline & & $\begin{array}{l}\text { \% profissionais enviando o resumo de } \\
\text { alta hospitalar para os diferentes níveis } \\
\text { de atenção }\end{array}$ & 3,00 & 3 & $\begin{array}{l}100,0 \\
\text { implantado }\end{array}$ & \\
\hline & \multirow[t]{2}{*}{$\begin{array}{l}\text { Uso da infor- } \\
\text { mação }\end{array}$} & $\begin{array}{l}\text { \% profissionais utilizando as Informa- } \\
\text { ções referenciadas entre os níveis de } \\
\text { atenção para a tomada de decisão na } \\
\text { conduta clínica }\end{array}$ & 0,00 & 5 & $\begin{array}{l}0,0 \\
\text { não implantado }\end{array}$ & \multirow{2}{*}{ 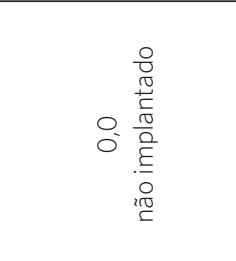 } \\
\hline & & $\begin{array}{l}\text { \% profissionais dos três níveis de aten- } \\
\text { ção monitorando e discutindo os indica- } \\
\text { dores epidemiológicos }\end{array}$ & 0,00 & 5 & $\begin{array}{l}0,0 \\
\text { não implantado }\end{array}$ & \\
\hline
\end{tabular}

Fonte: Elaboração própria.

Dentre os subcomponentes da 'coordenação da gestão clínica' o que apresentou melhor resultado foi o 'seguimento do paciente' (44,6\%), seguido da 'coerência da atenção' (19,0\%), classificados como de implantação incipiente e não implantado, respectivamente. No 'seguimento do paciente', destacou-se a realização das ações de busca ativa de contatos e de faltosos (100,0\%). Os principais pontos observados foram a inexistência de uma equipe multidisciplinar para o tratamento e acompanhamento do paciente TB-MDR no nível terciário e o percentual de profissionais que referiram a existência de um responsável clínico para coordenar o seguimento do paciente $(58,1 \%)$. Na atenção primária, um pequeno percentual de médicos referiu consultar especialistas para retirar dúvidas sobre o seguimento dos pacientes $(23,5 \%)$ e realizar a consulta de seguimento do paciente TB-MDR (36,8\%). Apenas $12,9 \%$ dos médicos e enfermeiros relataram realizar acompanhamento dos casos internados (quadro 4). 
Quadro 4. Resultado do componente 'coordenação da gestão clínica' entre os níveis de atenção ao paciente com tuberculose multidrogarresistente. Recife, 2015

\begin{tabular}{|c|c|c|c|c|c|c|}
\hline Componente & Subcomponente & Indicador & $\begin{array}{l}\text { Pontuação } \\
\text { Obtida }\end{array}$ & $\begin{array}{l}\text { Pontuação } \\
\text { Máxima }\end{array}$ & $\begin{array}{l}\text { Resultado (\%) e clas- } \\
\text { sificação por indicador }\end{array}$ & $\begin{array}{l}\text { Resultado (\%) e } \\
\text { classificação por } \\
\text { subcomponente }\end{array}$ \\
\hline \multirow{16}{*}{ 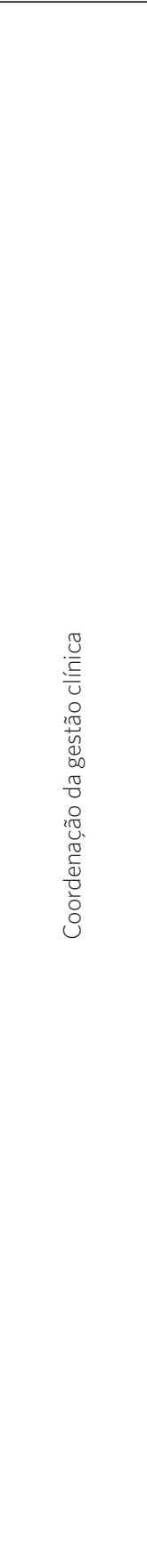 } & $\begin{array}{l}\text { Seguimento do } \\
\text { paciente }\end{array}$ & Realização do TDO & 1,16 & 3 & $\begin{array}{l}38,7 \\
\text { implantação incipiente }\end{array}$ & \multirow{8}{*}{ 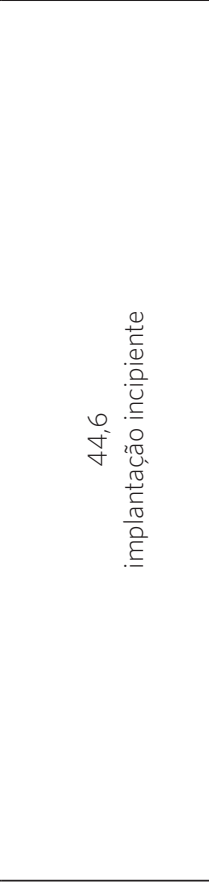 } \\
\hline & & $\begin{array}{l}\text { Existência de uma equipe multidisciplinar } \\
\text { composta por enfermeiro, médico, as- } \\
\text { sistente social, técnico de enfermagem, } \\
\text { farmacêutico, bioquímico, nutricionista e } \\
\text { psicólogo no nível terciário }\end{array}$ & 0,00 & 3 & $\begin{array}{l}0,0 \\
\text { não implantado }\end{array}$ & \\
\hline & & $\begin{array}{l}\text { Existência de responsável clínico para coor- } \\
\text { denar o seguimento do paciente }\end{array}$ & 2,32 & 4 & $\begin{array}{l}58,1 \\
\text { implantação parcial }\end{array}$ & \\
\hline & & $\begin{array}{l}\text { \% profissionais médicos do primeiro nível } \\
\text { que consultam o médico especialista para } \\
\text { tirar as dúvidas sobre o seguimento dos } \\
\text { pacientes }\end{array}$ & 0,00 & 3 & $\begin{array}{l}0,0 \\
\text { não implantado }\end{array}$ & \\
\hline & & $\begin{array}{l}\text { \% profissionais médicos da atenção primá- } \\
\text { ria que realizam a consulta de seguimento } \\
\text { do paciente TB-MDR }\end{array}$ & 0,00 & 3 & $\begin{array}{l}0,0 \\
\text { não implantado }\end{array}$ & \\
\hline & & $\begin{array}{l}\% \text { profissionais realizando acompanhamen- } \\
\text { to dos casos internados }\end{array}$ & 0,00 & 3 & $\begin{array}{l}0,0 \\
\text { não implantado }\end{array}$ & \\
\hline & & $\begin{array}{l}\text { \% profissionais dos três níveis de atenção } \\
\text { que investigam os contatos e realizam } \\
\text { seguimento clínico }\end{array}$ & 3,00 & 3 & $\begin{array}{l}100,0 \\
\text { implantado }\end{array}$ & \\
\hline & & $\begin{array}{l}\text { \% profissionais dos três níveis de atenção } \\
\text { que realizam busca dos faltosos }\end{array}$ & 3,00 & 3 & $\begin{array}{l}100,0 \\
\text { implantado }\end{array}$ & \\
\hline & \multirow[t]{8}{*}{$\begin{array}{l}\text { Coerência da } \\
\text { atenção }\end{array}$} & $\begin{array}{l}\text { \% pacientes com medicamentos preco- } \\
\text { nizados disponíveis para o tratamento da } \\
\text { TB-MDR }\end{array}$ & 3,00 & 3 & $\begin{array}{l}100,0 \\
\text { implantado }\end{array}$ & \multirow{8}{*}{ 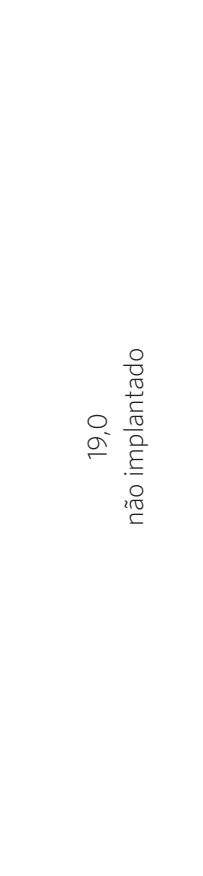 } \\
\hline & & $\begin{array}{l}\text { \% pacientes realizando todos os exames } \\
\text { preconizados para diagnóstico e acompa- } \\
\text { nhamento, como baciloscopia de escarro, } \\
\text { cultura de secreção, raio-x e teste anti-HIV }\end{array}$ & 0,00 & 3 & $\begin{array}{l}0,0 \\
\text { não implantado }\end{array}$ & \\
\hline & & $\begin{array}{l}\text { Existência de um protocolo padronizado } \\
\text { entre níveis para o acompanhamento e } \\
\text { tratamento do paciente TB-MDR }\end{array}$ & 1,45 & 3 & $\begin{array}{l}48,3 \\
\text { implantação incipiente }\end{array}$ & \\
\hline & & $\begin{array}{l}\text { \% profissionais que conhecem e utilizam } \\
\text { protocolo para a conduta clínica relativa a } \\
\text { diagnóstico, tratamento, exames e critérios } \\
\text { de encaminhamento para outro nível de } \\
\text { atenção entre níveis }\end{array}$ & 0,00 & 3 & $\begin{array}{l}0,0 \\
\text { não implantado }\end{array}$ & \\
\hline & & $\begin{array}{l}\text { \% profissionais realizando discussão dos } \\
\text { casos clínicos entre os níveis de atenção }\end{array}$ & 0,00 & 4 & $\begin{array}{l}0,0 \\
\text { não implantado }\end{array}$ & \\
\hline & & $\begin{array}{l}\text { \% profissionais realizando matriciamento } \\
\text { dos casos entre os níveis atenção }\end{array}$ & 0,00 & 3 & $\begin{array}{l}0,0 \\
\text { não implantado }\end{array}$ & \\
\hline & & $\begin{array}{l}\text { Capacitação conjunta realizada entre os } \\
\text { profissionais dos níveis de atenção }\end{array}$ & 0,29 & 3 & $\begin{array}{l}9,7 \\
\text { não implantado }\end{array}$ & \\
\hline & & $\begin{array}{l}\text { \% profissionais dos três níveis de atenção } \\
\text { investigando os efeitos colaterais }\end{array}$ & 0,00 & 3 & $\begin{array}{l}0,0 \\
\text { não implantado }\end{array}$ & \\
\hline
\end{tabular}

Fonte: Elaboração própria. 
A 'coerência da atenção' obteve o pior resultado entre os subcomponentes avaliados $(19,0)$. $\mathrm{O}$ acesso dos pacientes aos medicamentos preconizados para o tratamento da TB-MDR apresentou o melhor resultado (implantado), pois $80,6 \%$ dos profissionais relataram não haver problemas na dispensação. Dificuldades foram relatadas com relação à existência de padronização na atenção do paciente com TB-MDR; apenas 48,4\% dos profissionais referiram existir protocolo padronizado entre níveis de atenção para o acompanhamento e seguimento desses pacientes, ao passo que apenas $38,7 \%$ conhecem e utilizam o protocolo para a conduta clínica (quadro 4).

Algumas fragilidades foram observadas nas ações de educação permanente, tais como a baixa participação dos profissionais em capacitações conjuntas com profissionais de outros níveis de atenção (9,7\%) e a ausência de discussão e matriciamento dos casos clínicos entre os diferentes níveis de atenção. Além desses problemas, se destacaram o baixo percentual de pacientes que realizaram todos os exames preconizados (11,1\%) e a baixa investigação de efeitos colaterais pelos profissionais dos três níveis de atenção (45,2\%) (quadro 4).

\section{Discussão}

Neste estudo, observou-se que os níveis de atenção ao paciente com TB-MDR em Recife organizam-se de forma separada, com falhas na interligação da rede de serviços, resultando numa assistência descoordenada e fragmentada. Tal realidade se deve a problemas na utilização dos mecanismos de coordenação e à descontinuidade no fluxo de informação, ou seja, devido à discordância com os atributos de coordenação da atenção. Porém, ressalta-se que um dos objetivos finais das redes de atenção é melhorar a eficiência global na prestação e continuidade dos cuidados por meio de um objetivo intermediário, que é a coordenação dos serviços ${ }^{8}$.

Cabe destacar que este estudo apresentou algumas limitações, uma vez que pesquisas nacionais sobre a coordenação do cuidado ainda são escassas, dificultando a comparação com os resultados encontrados, o que demanda a utilização de estudos realizados fora do Brasil. Adicionalmente, entende-se que a abordagem aos pacientes poderia oferecer informações complementares sobre a coordenação do cuidado. No entanto, ao entrevistar profissionais nos três níveis de atenção, a intenção era a de ampliar a confiabilidade das informações.

A existência de fluxos assistenciais definidos para o paciente TB-MDR sem, contudo, um funcionamento adequado acarreta obstáculos no acesso aos serviços, como barreiras físicas e burocráticas. Esse acesso deve garantir ao paciente qualidade e resolubilidade na sua assistência em todos os níveis de atenção ${ }^{\mathbf{1 7}}$.

As condições de vida e trabalho expõem a população a diferentes riscos de desenvolver a tuberculose. Estudos revelam a baixa escolaridade, a dificuldade em relação ao transporte coletivo para se deslocar até as unidades de saúde, a demora na espera pela consulta e a baixa renda como fatores de aumento da vulnerabilidade das pessoas ${ }^{\mathbf{1 8}}$. Outros incentivos para adesão dos pacientes ao tratamento da tuberculose são a disponibilidade de benefícios tais como cesta básica mensal, cafés da manhã e vale transporte ${ }^{19}$.

Além disso, a Organização Mundial da Saúde (OMS) declara que a principal causa para a gravidade da situação da tuberculose no mundo é a desigualdade social e que os bolsões de pobreza das cidades mais populosas constituem terreno fértil para a disseminação da doença $a^{6,17,18}$. Diante dessa vulnerabilidade social, torna-se fundamental a garantia de benefícios que assegurem a adesão e a conclusão do tratamento ${ }^{18}$, especialmente porque ajudam no combate à determinação social da doença.

Para garantir que a coordenação da informação ocorra de forma efetiva, os profissionais dos diferentes níveis de atenção têm que, além de registrar as informações, transferi-las e utilizá-las de maneira adequada. Assim, o preenchimento adequado dos instrumentos de 
informação referentes aos mecanismos formais envolvidos no atendimento ao paciente e que garantem a continuidade do cuidado, é atributo para que ocorra a coordenação da informação. A alimentação oportuna do SITETB é pré-requisito para que os pacientes recebam a medicação em tempo oportuno, influenciando diretamente na coordenação da gestão clínica, que tem como um dos seus atributos a prescrição correta e sem discordâncias dos medicamentos preconizados pelos profissionais dos diferentes níveis de atenção"

O não preenchimento das fichas de referência e contrarreferência pode configurar um obstáculo para a continuidade da assistência ao paciente ${ }^{19}$. Nesse aspecto, o resultado deste estudo está em consonância com a pesquisa realizada por Assis et al. ${ }^{7}$, que identificou que as equipes dos serviços não garantem o registro das informações necessárias para os mecanismos de referência e contrarreferência no atendimento ao doente com tuberculose. Estudo realizado por Silva et al. ${ }^{20}$ também identificou problemas de preenchimento nas guias de referência tais como a falta de dados importantes para definição do diagnóstico e resolução dos problemas apresentados.

Para que exista coordenação da informação é necessário tanto transmiti-la entre os níveis, quanto utilizá-la na tomada de decisão9,10. Ainda sobre o uso da informação, é necessário destacar a importância do acompanhamento dos indicadores epidemiológicos, uma vez que a recomendação do PCT é conhecer a magnitude da doença por seus dados de morbidade e mortalidade, sua distribuição, fatores de risco e tendência no tempo. Essa prática é fundamental para o planejamento das ações de controle e acompanhamento dos casos, embora muitas vezes fica subjugada como uma atividade sem sentido e de cunho burocrático dos sistemas de informação devido à obrigatoriedade desse processo para o repasse de verbas oriundas do Ministério da Saúde ${ }^{\mathbf{9}, 20}$.

A existência de uma equipe multidisciplinar favorece o atendimento integral ao paciente e uma assistência coordenada ${ }^{5}$. Além disso, estudos revelam que o médico, seja da atenção primária ou especialista, capaz de coordenar o seguimento do paciente é um fator imprescindível para a coordenação da assistência. No entanto, ressalta-se que, no Brasil, o médico não é o único responsável pelo acompanhamento desses pacientes. Apesar disso, os resultados deste estudo revelam que nenhum dos médicos e enfermeiros entrevistados se considera coordenador do cuidado. Um dos atributos para a coordenação da atenção é a existência desse responsável clínico que atue entre os níveis de atenção e que, de preferência, seja um profissional da atenção primária, já que ela deve atuar como coordenadora do cuidado $4,17,19$.

O PCT recomenda que os paciente TB-MDR sejam acompanhados pelos profissionais da atenção básica ${ }^{5,7}$. Este estudo constatou que poucos médicos consultam especialistas para retirar as dúvidas sobre o seguimento dos pacientes. Essa descontinuidade do fluxo de comunicação e informação pode se configurar em obstáculo à continuidade da assistência ${ }^{19}$.

O subcomponente coerência da atenção obteve um dos piores resultados entre todos os avaliados. As principais dificuldades dizem respeito à existência de padronização na atenção do paciente com TB-MDR, uma vez que poucos profissionais referiram existir protocolo entre níveis de atenção para o acompanhamento e seguimento desses pacientes. Contatou-se, também, um baixo percentual de profissionais que conheciam e utilizavam o protocolo para a conduta clínica. Um dos atributos da coerência da atenção para garantia de uma assistência coordenada é a implantação de mecanismos tais como guias de práticas clínicas compartilhadas, guias farmacológicos e guias de referência e contrarreferência adequadas ${ }^{1,17}$.

Sobre as ações de educação permanente, estudo realizado em municípios brasileiros mostrou que as capacitações para desenvolvimento de habilidades ou competências técnicas em áreas específicas são consideradas importantes para aumentar a resolutividade 
das equipes e que os gestores e gerentes compreendem que a formação continuada deve ter como base o processo de trabalho ${ }^{\mathbf{1 0} 11}$.

O matriciamento pode ser uma estratégia para promover interlocução entre distintos equipamentos da rede de serviços de saúde. Destaca, dentre as inovações mencionadas para qualificar as ações da ESF, que especialistas apoiem profissionais da atenção primária por meio de interconsulta, discussão de casos clínicos e capacitações ${ }^{21}$.É importante salientar também que o 'Manual de recomendações para controle da tuberculose no Brasil' preconiza a discussão de casos entre os profissionais dos diferentes níveis de atenção ${ }^{5}$.

É evidente que a atenção primária exerce papel central na constituição das redes de atenção à saúde, o que não é diferente na atenção ao paciente TB-MDR. No entanto, da mesma forma que não existe rede sem atenção primária robusta capaz de coordenar o cuidado, a atenção primária não consegue exercer seu papel sem um arranjo sólido e uma articulação virtuosa entre os níveis de atenção. Ressalta-se que uma fraca coordenação do cuidado é um importante obstáculo para a garantia da integralidade, do acesso e da oferta de serviços de saúde de qualidade ${ }^{9-11}$.

\section{Considerações finais}

Este estudo permitiu identificar fragilidades e lacunas em importantes componentes da coordenação do cuidado ao paciente com TB-MDR, permitindo o direcionamento de estratégias para sua melhoria. No entanto, é preciso reconhecer a complexidade desse cenário, uma vez que a tuberculose pode ser considerada um traçador da determinação social da doença, pois, de certo modo, as dificuldades históricas no seu enfrentamento expõem a iniquidade presente na sociedade ${ }^{11}$.

$\mathrm{O}$ adequado funcionamento dos fluxos assistenciais e do registro das informações necessárias para garantia da continuidade da assistência ao paciente é um aspecto importante que precisa ser considerado. Para tal, a organização da referência e contrarreferência mediante a implantação de um instrumento que garanta o compartilhamento das informações entre as unidades dos níveis de atenção primário, secundário e terciário pode ser uma estratégia a ser seguida, bem como o estabelecimento de um responsável clínico para coordenar o seguimento do paciente entre os níveis.

É importante destacar tanto o papel fundamental da organização dos serviços de saúde na detecção e tratamento dos casos de tuberculose como a coordenação como instrumento determinante nesse processo. A TB-MDR é um problema crescente, sendo a mais prevalente das doenças com resistência antimicrobiana ${ }^{22}$. Portanto, é imprescindível que se enfrente esse desafio.

\section{Colaboradores}

Barros DBM (0000-0002-7681-9923)* contribuiu para a coleta e análise dos dados, concepção e planejamento da pesquisa, bem como para a redação do manuscrito. Costa JMBS (0000-0002-5809-4156)* e Reis YAC (0000-0002-5883-496X)* contribuíram igualmente para a concepção e o planejamento da pesquisa, bem como revisão e aprovação da versão final do manuscrito. Albuquerque AC (0000-0001-6305-4127)* contribuiu para a análise dos dados, redação e aprovação final do manuscrito. Felisberto E (0000-00022316-2251)* contribuiu para a concepção da pesquisa, revisão e aprovação da versão final do manuscrito. 


\section{Referências}

1. Vázquez ML, Vargas I, Unger JP, et al. Evaluating the effectiveness of care integration strategies in different healthcare systems in Latin America: the EQUITY-LA II quasi-experimental study protocol. BMJ Open. 2015; 5(7):e007037.

2. Mendes EV. As redes de atenção à saúde. 2. ed. Brasília, DF: Ministério da Saúde; OPAS; 2011.

3. Starfield B. Atenção primária: equilíbrio entre necessidades de saúde, serviços e tecnologia. Brasília, DF: Unesco; Ministério da Saúde; 2002.

4. Terraza NR. Coordinación y Continuidad: Un marco para el análisis [tesina máster] [internet]. Barcelona: Universitat Pompeu Fabra; 2004.

5. Brasil. Ministério da Saúde, Secretaria de Vigilância em Saúde, Departamento de Vigilância Epidemiológica. Manual de recomendações para o controle da tuberculose no Brasil. Brasília, DF: Ministério da Saúde; 2011.

6. Word Health Organization. Global status reporton non-communicable diseases. Geneva: WHO; 2014.

7. Assis EG, Beraldo AA, Monroe AA, et al. A coordenação da assistência no controle da tuberculose. Rev. Esc. Enferm. USP; 2012; 46(1):111-118.

8. Vargas I, Mogollón-Pérez AS, De Paepe P, et al. Barriers to healthcare coordination in market-based and decentralized public health systems: a qualitative study in healthcare networks of Colombia and Brazil. Health Policy Plan. 2016; 31(6):736-48.

9. Andrade HS, Oliveira VC, Gontijo TL, et al. Avaliação do Programa de Controle da Tuberculose: um estudo de caso. Saúde debate. 2017; 41(esp):242-58.

10. Bousquat A, Giovanella L, Campos EMS, et al. Atenção primária à saúde e coordenação do cuidado nas regiões de saúde: perspectiva de gestores e usuários. Ciênc. Saúde Colet. 2017; 22(4):1141-54.
11. Cunha NV, Cavalcanti MLT, Santos MLF, et al. Estrutura, organização e processos de trabalho no controle da tuberculose em municípios do estado do Rio de Janeiro, RJ, Brasil. Interface (Botucatu). 2015; 19(53):251-264.

12. Yin RK. Estudo de Caso - Planejamento e Métodos. 5. ed. Porto Alegre: Bookman; 2015.

13. Brasil. Ministério da Saúde, Secretaria de Vigilância em Saúde. Boletim Epidemiol. 2018: (49):1-14.

14. Recife. Secretaria Municipal de Saúde. Plano Plurianual 2014 -2017. Recife: Secretaria Municipal de Saúde; 2014.

15. Reid R, Haggerty J, Mckendry R. Defusing the confusion: concepts and measures of continuity of healthcare. Ottawa: Canadian Health Services Research Foundation; 2002.

16. Reis YAC, Cesse EAP, Carvalho EF. Consensos sobre o papel do gestor estadual na regionalização da assistência à saúde no Sistema Único de Saúde (SUS). Rev Bras Saúde Matern Infant. 2010; 10(supl1):15772 .

17. Vargas I, Mogollon-Perez AS, De Paepe P, et al. Do existing mechanisms contribute to improvements in care coordination across levels of care in health services networks? Opinions of the health personnel in Colombia and Brazil. BMC Health Services Research. 2015; 29(15):213.

18. Pinheiro RS, Oliveira GP, Oliveira EXG, et al. Determinantes sociais e autorrelato de tuberculose nas regiões metropolitanas conforme a Pesquisa Nacional por Amostra de Domicílios, Brasil. Rev Panam Salud Publica. 2013; 34(6):446-51.

19. Ireson CL, Slavova SS, Steltenkamp CL, et al. Bridging the care continuum: patient information needs for specialist referrals. BMC Health Serv Res. 2009; 9(163)1-27. 
20. Silva EM, Assis MMA, Villa TCS, et al. Coordenação dos serviços de atenção primária em saúde no controle da tuberculose em um município da Bahia, Brasil. Rev. Baiana Saúde Publ. 2010; 34(2):227.

21. Almeida PF, Fausto MCR, Giovanella L. Fortalecimento da atenção primária à saúde: estratégia para potencializar a coordenação dos cuidados. Rev Panam Salud Publica. 2011; 29(2):84-95.
22. World Health Organization. The end TB strategy [internet]. Geneva: World Health Organization; 2015. [acesso em 2019 maio 4]. Disponível em: http://www. who.int/tb/End_TB_brochure.pdf?ua=1.

Recebido em 06/05/2019

Aprovado em 20/01/2020

Conflito de interesses: inexistente

Suporte financeiro: não houve 